\title{
Psychiatric Formulation and the Structural Determinants of Mental Health
}

\author{
Allison M. Bailey ${ }^{1}$ (D) \\ Received: 15 February 2020 / Accepted: 8 September 2020 / Published online: 17 September 2020 \\ (C) Academic Psychiatry 2020
}

One of my first patients of residency, a high-achieving high school student, presented with debilitating anxiety about multiple topics ranging from academic performance to social acceptance. Her chart history suggested a diagnosis of generalized anxiety disorder. When I met her, I listened carefully to her personal narrative and interpreted each anecdote through the lens of my initial diagnostic theory.

Midway through the interview she revealed her concern that her father would get pulled over and shot while driving home from work. This was 2019 in Baltimore City, in the notso-distant wake of the death of Freddie Gray and protest movements around the country surrounding police violence against Black people. I fumbled through the remainder of my patient's interview, distracted by the realization that my diagnostic reasoning skills might be insufficient to adequately formulate her mental suffering.

At my home institution trainees are taught to understand mental suffering through a diagnostic model called the Perspectives of Psychiatry, which was articulated in a 1983 publication by two psychiatrists from Johns Hopkins Hospital [1]. The Perspectives model considers four factors that shape psychiatric presentations: disease, dimension, behavior, and life story. Each factor is associated with a specific therapeutic intervention. Diseases can be diagnosed based on clinical syndromes and treated with medications. Dimensional vulnerabilities or problematic personality traits can be addressed through psychotherapy. Harmful behavioral patterns such as substance use can be interrupted through rehabilitation. Life stories informed by maladaptive interpretations of events and relationships can be rescripted - this approach can help a patient gain a sense of mastery over her circumstances by adopting a new mindset rather than changing the external environment.

Allison M. Bailey

ab@jhmi.edu

1 The Johns Hopkins University School of Medicine, Baltimore, MD, USA
For me, the Perspectives model functions as a system of four complimentary lenses that allows me to view my patients' narratives in totality. I think about each patient through all four Perspectives, working to understand the relative contribution of each one to an individual's mental suffering. For my patient with generalized anxiety disorder, I might consider whether she has a disease amenable to medical treatment or anxious dimensional traits amenable to therapy. I might ask whether she is engaging in behaviors that exacerbate anxiety, such as caffeine use. Finally, I might examine her life story for elements related to anxiety such as academic stress and then help her rescript these elements into a more empowering personal narrative.

In addition to the Perspectives model, I have access to many other resources such as psychological assessments, laboratory tests, and neuroimaging. However, as I progress through my training I often encounter illness presentations that I struggle to formulate despite the expansive clinical toolkit at my disposal.

My patient with generalized anxiety disorder feared for her father's death following years of exposure to examples of police violence against Black people. At its core, her illness presentation reflects the devastating mental health impacts of structural racism. This patient's experience is not uncommon. Many of my patients present with personal narratives that illustrate, either implicitly or explicitly, the complex relationships between structural forces and mental health. I have met parents who fear for the safety of their children in the setting of ongoing community violence. I have met young adults who mourn the loss of siblings and friends to high rates of violent crime. I recall one patient who struggled to establish a personal identity following decades of incarceration for a minor offense. The structural forces underlying these narratives are not addressed in the DSM-5. They are not diseases I can treat with medicine, behavioral patterns I can interrupt, dimensional vulnerabilities amenable to therapy, or life stories I can rescript in a therapeutic way. At the same time, it feels irresponsible to recognize these limitations without reaching for something more.

In trying to clarify what felt missing from my training, I encountered the conceptual framework of structural 
competency. This domain of medicine encourages practitioners to shift our gaze above the level of the individual encounter to recognize how institutions and policies influence the health of our patients [2]. For psychiatry specifically, structural competency encourages practitioners to "bridge the micro-processes of their interactions with patients with the macro-processes of population level inequalities that often determine their patients' mental health outcomes" [3]. Is structural competency the missing piece from my diagnostic toolkit that would teach me to think more broadly about my patients' lived experiences?

I work in a city where powerful structural forces shape the experience of mental illness for thousands of individuals. I should explore these themes with my patients, especially when they are woven explicitly into the threads of their narratives. When I develop diagnostic formulations, I should consider how my patients' choices are constrained by access to employment, transportation, pharmacies, and information. Specialties such as public health and political science are dedicated to this type of work, but I cannot refer my patients to these specialties to heal their mental suffering. I must be equipped to listen, learn, and address these themes within my own clinical practice.

Some believe that structural competency falls under the domain of life story reasoning within the Perspectives model. How, then, can we use life story reasoning to understand structural determinants of mental health? How can we rescript narratives involving structural forces in ways that are therapeutic for our patients? When mental suffering arises from challenges that are engineered and perpetuated by society, the narrative is merely a reflection of a problem more powerful than any one of us.

Social injustice and its impacts on health outcomes are not new crises. However, the murders of George Floyd, Breonna
Taylor, and Ahmaud Arbery have reemphasized their critical importance. Physicians across specialties are reexamining the ways by which medical institutions perpetuate structural racism and reinforce healthcare disparities [4, 5]. Psychiatrists must do the same. The frameworks we use to formulate psychiatric illness cannot exclude the impacts of structural racism and other manifestations of social injustice. While we cannot solve these crises within a single patient encounter, we can broaden our competencies to move towards a more just model of patient care.

Acknowledgment The author wishes to thank Dr. Hal Kronsberg for his thoughtful review of this manuscript.

\section{Compliance with Ethical Standards}

Disclosure The author states that there is no conflict of interest.

\section{References}

1. McHugh P, Slavney P. The perspectives of psychiatry. Baltimore: The Johns Hopkins University Press; 1983.

2. Metzl J, Hansen H. Structural competency: theorizing a new medical engagement with stigma and inequality. Soc Sci Med. 2014;103: 126-33.

3. Metzl J, Hansen H. Structural competency and psychiatry. JAMA Psychiat. 2018;75(2):115-6.

4. Evans M, Rosenbaum L, Malina D, Morrissey S, Rubin E. Diagnosing and treating systemic racism. New Engl J Med. 2020;383:274-6.

5. Cénat J. How to provide anti-racist mental health care. Lancet Psychiatry. 2020. https://doi.org/10.1016/S2215-0366(20)30309-6.

Publisher's Note Springer Nature remains neutral with regard to jurisdictional claims in published maps and institutional affiliations. 